\title{
On the Reversed Average Intensity Order
}

\author{
Majid Rezaei* and Vahideh Ahrari Khalef \\ Birjand University
}

\begin{abstract}
Based on the increasing property of ratio of average reversed hazard rates of two non-negative random variables a new stochastic order for the sake of comparison of two lifetime distributions is proposed. This stochastic order admits some distinguishing properties. In order to illustrate the obtained results, a semi-parametric model which is called reverse hazard power and a mixture model of proportional reversed hazards are taken into account. Some examples are given to explain some facts.
\end{abstract}

Keywords. Reversed hazard rate; stochastic order; mixture; reversed hazard power.

MSC 2010: 60E15.

\section{Introduction and Preliminaries}

The concepts of stochastic orders and aging notions are very useful in different branches of sciences such as biology, social sciences, economics and statistics. As two probability distributions are ordered according to a special partial order some information may then be provided. For example for two lifetime devises, a particular stochastic order may determine that which one of two devices ages faster. From other view, to measure various quantities in two distributions several biometric functions have been defined by which the partial orders are constructed. One of these functions is the well known reversed hazard rate (RHR) function. If density function exists then the RHR function is defined as the ratio of the density to the distribution function. We refer to Gupta and Nanda (2001), Finkelstein (2002), Shaked

\footnotetext{
* Corresponding author
} 
and Shanthikumar (2007) and Brito et al. (2011) for some details about ordering properties of the RHR function. Recently some researchers proposed orderings between two models through monotonicity property of ratio of failure rate and mean residual life of two life distributions (cf. Kalashnikov and Rachev, 1986 and Finkelstein, 2006). Recently, Rezaei et al. (2014) proposed a new stochastic order based on the increasing property of ratio of two RHR functions.

In the context of reliability and life testing, Jiang et al. (2003) proposed a quantitative measure, called Aging Intensity (AI) function, defined as the ratio of the hazard rate (HR) $r(t)=f(t) / \bar{F}(t)$ to a baseline hazard rate $H(t)$. One natural choice for $H(t)$ is the average hazard rate $t^{-1} \int_{0}^{t} r(x) d x$, for all $t>0$. Formally, the AI function is

$$
L(t)=\frac{r(t)}{\frac{1}{t} \int_{0}^{t} r(x) d x}, \quad \text { for any } t>0 .
$$

Recently, Nanda et al. (2007) investigated some useful stochastic and aging properties of the AI function. In their paper, they defined a new stochastic order based on the comparison of the AI functions of two lifetime variables. Denote by $r_{i}$ the HR of $X_{i}$, for $i=1,2$. Nanda et al. (2007) said that the random variable $X_{1}$ is smaller than the random variable $X_{2}$ in average intensity order (denoted by $X_{1} \leqslant A I X_{2}$ ), if $L_{1}(t) \geqslant L_{2}(t)$, for all $t \geqslant 0$, where $L_{i}(t)=t r_{i}(t) / \int_{0}^{t} r_{i}(x) d x, i=1,2$. For two non-negative random variables $X_{1}$ and $X_{2}$ with respective survival functions $\bar{F}_{1}$ and $\bar{F}_{2}$, in Theorem 3.1(iii) of their work it is proved that $X_{1} \leqslant A I X_{2}$ if and only if $\ln \bar{F}_{1}(x) / \ln \bar{F}_{2}(x)$ is increasing in $x>0$. In this paper, we are going to define a dual stochastic order for the AI order in the sense that we use the well known RHR function instead of the HR function. We shall name this order as reversed average intensity (RAI) order. Relationships among the RAI order and other well known stochastic orders along with some aging properties are first investigated. Preservation properties of the new stochastic order under increasing transformations and under the formation of parallel systems are then obtained. To compare two mixture models of proportional reversed hazard rates (PRHR) that is essentially due to $\mathrm{Li}$ and $\mathrm{Li}$ (2008), we make use of the new stochastic order. Among the results, motivations for the new order are explained by some examples.

Let $X_{1}$ and $X_{2}$ be two non-negative random variables with respective supports $S_{1}=\left(l_{1}, u_{1}\right)$ and $S_{2}=\left(l_{2}, u_{2}\right)$, having absolutely continuous distribu- 
tion functions $F_{1}$ and $F_{2}$ and probability density functions (pdf) $f_{1}$ and $f_{2}$, respectively. Further, assume that $q_{1}$ and $q_{2}$ are the RHR's of $X_{1}$ and $X_{2}$, which are, respectively, given by

$$
q_{1}(x)=\frac{f_{1}(x)}{F_{1}(x)} \text { and } q_{2}(x)=\frac{f_{2}(x)}{F_{2}(x)}, \quad \forall x>0 .
$$

In the following, two stochastic orders are given according to Shaked and Shanthikumar (2007).

Definition 1. The random variable $X_{1}$ is said to be smaller than $X_{2}$ in usual stochastic order (denoted by $X_{1} \leqslant S T X_{2}$ ) if $F_{1}(x) \geqslant F_{2}(x)$, for all $x \geqslant 0$.

Definition 2. It is said that $X_{1}$ is smaller than $X_{2}$ in the sense of RHR order (denoted by $X_{1} \leqslant R H R X_{2}$ ) if, $q_{1}(x) \leqslant q_{2}(x)$, for all $x>0$, or equivalently, if $F_{2}(x) / F_{1}(x)$ is increasing in $x \in(0, \infty)$.

The following definition can be found in Nanda et al. (2003).

Definition 3. The random variable $X_{1}$ is said to have decreasing reversed hazard rate (DRHR) property, if $q_{1}(x)$ is decreasing in $x>0$, or equivalently if $F_{1}$ is log-concave.

Definition 4. A non-negative real valued bivariate function $h$ is said to be totally positive of order 2 (abbreviated by $\mathrm{TP}_{2}$ ) in $(x, y) \in \chi \times \gamma$, whenever $h\left(x_{1}, y_{1}\right) h\left(x_{2}, y_{2}\right) \geqslant h\left(x_{1}, y_{2}\right) h\left(x_{2}, y_{1}\right)$, for all $x_{1} \leqslant x_{2} \in \chi$ and $y_{1} \leqslant y_{2} \in \gamma$, where $\chi$ and $\gamma$ are two arbitrary subsets of the real line $R$.

Note that $h_{i}(t)$ is said to be $\mathrm{TP}_{2}(i, t)$, in $i=1,2$ and $t \in(0, \infty)$ whenever the function $h:(i, t) \mapsto h_{i}(t)$ is $\mathrm{TP}_{2}$ in $(i, t) \in \chi \times \gamma$, with $\chi=\{1,2\}$ and $\gamma=(0, \infty)$.

Suppose that $V_{1}$ and $V_{2}$ are two non-negative random variables and $F_{0}$ is a distribution function on $(0, \infty) . G_{i}(x)=E\left(F_{0}^{V_{i}}(x)\right), i=1,2$ is a distribution function over $(0, \infty)$. The random variables $V_{1}$ and $V_{2}$ are called mixing random variables, $F_{0}$ is called baseline population and $G_{i}$ the overall population. $\mathrm{Li}$ and $\mathrm{Li}$ (2008) investigated the conditions under which a particular type of a stochastic order between mixing variables implies the same stochastic 
order of their corresponding overall populations. We study this topic for our proposed stochastic order. We refer also the reader to Li and Zhao (2011) and $\mathrm{Xu}$ and $\mathrm{Li}$ (2008) for some analogous results regarding some other mixture models. Throughout the paper, increasing and decreasing mean nondecreasing and non-increasing, respectively. It is implicity assumed that all the integrations, derivatives and expectations exist whenever they are appeared. Recall that a series system with $n$ components works when all of their components work and a parallel system with $n$ components works when at least one of their components works. That is if $X_{1}, X_{2}, \ldots, X_{n}$ denote the life lengths of $n$ components including in a system, then the life length of the system under the series and the parallel structures are $\min \left\{X_{1}, X_{2}, \ldots, X_{n}\right\}$ and $\max \left\{X_{1}, X_{2}, \ldots, X_{n}\right\}$, respectively.

\section{Motivations, Main Definitions and Basic Prop- erties}

In this section, we first give some motivations for the study of the RAI order. Then, we obtain some distinguishing properties of this stochastic order including a number of relationships between this order and the other stochastic orders. Some preservation and characterization results regarding monotonic transformations and series or/and parallel systems are given. First of all, we propose the reversed aging intensity function as a dual measure for the AI function given in (1). Likewise the AI function which considers the left tail of distributions, the RAI function serves as a quantitative measure that takes the right tails of distributions into account. Suppose that $X$ is a non-negative random variable with distribution function $F$, and with support $S_{X}=\left(l_{X}, u_{X}\right)$, where $l_{X}=\inf \{x: F(x)>0\}$ and $U_{X}=\sup \{x: F(x)<1\}$. By assuming that $X$ is a bounded random variable in the sense that $u_{X}<\infty$, the RAI function of $X$ is defined by

$$
L^{*}(t)=\frac{q(t)}{\frac{1}{u_{X}-t} \int_{t}^{u_{X}} q(x) d x}, \quad \text { for any } t<u_{X},
$$

where $q$ is the RHR function of $X$. Let the non-negative random variable $X_{i}$ with RHR function $q_{i}$, and with support $S_{i}=\left(l_{i}, u_{i}\right), i=1,2$ have the RAI

$$
L_{i}^{*}(t)=\frac{q_{i}(t)}{\frac{1}{u_{i}-t} \int_{t}^{u_{i}} q_{i}(x) d x}, \quad \text { for any } t<u_{i} .
$$


Now, assume that the upper bound $u_{i}$ of the random variable $X_{i}$, for each $i=1,2$ is finite. Then, denote by $\Lambda_{i}(x)$ the average of the RHR function of $X_{i}$ on the interval $\left(x, u_{i}\right)$ for $i=1,2$. It can be obtained, for any $t>0$, that

$$
\begin{aligned}
\Lambda_{i}(t) & =\frac{1}{u_{i}-t} \int_{t}^{u_{i}} q_{i}(x) d x \\
& =\frac{\ln F_{i}\left(u_{i}\right)-\ln F_{i}(t)}{u_{i}-t}=\frac{-\ln F_{i}(t)}{u_{i}-t}, \quad i=1,2 .
\end{aligned}
$$

When the upper bounds of the supports of $X_{1}$ and $X_{2}$ are not finite but they are equal, i.e. $u_{1}=u_{2}$, we can easily observe that the ratio $\Lambda_{2}(t) / \Lambda_{1}(t)$ is increasing for $t>0$, if and only if $L_{1}^{*}(t) \leqslant L_{2}^{*}(t)$, for all $t \in\left(0, u_{1}\right)$. It is noticeable here that once this ratio is increasing, it turns out that the average RHR of $X_{1}$ decreases faster with respect to the average RHR of $X_{2}$. This means that a device with lifetime $X_{1}$ ages faster, in some stochastic senses, than another device whose lifetime is $X_{2}$. The importance of such an investigation stems from the fact that it provides a concept of relative ageing of two probability distributions (see, e.g., Sengupta and Deshpande, 1994 and Lai and Xie, 2003). For the sake of comparison of two probability distributions according to their RAI functions, we define a new stochastic order as follows. At the continue we always assume that $u_{1}=u_{2}$.

Definition 5. We say that $X_{1}$ is smaller than $X_{2}$ in the RAI order (denoted by $\left.X_{1} \leqslant R A I X_{2}\right)$ whenever $L_{1}^{*}(x) \leqslant L_{2}^{*}(x)$, for all $x \in\left(0, u_{1}\right)$, or equivalently if $\frac{\ln F_{2}(x)}{\ln F_{1}(x)}$ is increasing in $x$ over $(0, \infty)$.

It can be easily seen that $X_{1} \leqslant_{R A I} X_{2}$ if and only if there exists a nonnegative increasing function $\beta(x)$ such that $F_{2}(x)=\left[F_{1}(x)\right]^{\beta(x)}$, for all $x>0$, that is the distribution function of the random variable $X_{2}$ is an exponentiated function of the distribution function of the random variable $X_{1}$. Moreover, one can see that $X_{1} \leqslant R A I X_{2}$ if and only if

$$
\frac{q_{1}(x)}{\int_{x}^{\infty} q_{1}(u) d u} \geqslant \frac{q_{2}(x)}{\int_{x}^{\infty} q_{2}(u) d u},
$$

for all $x>0$. The following definition is due to Rezaei et al. (2014).

Definition 6. The random variable $X_{1}$ is said to be smaller than $X_{2}$ in 
relative RHR order (denoted by $X_{1} \leqslant R R H X_{2}$ ), if $q_{2}(t) / q_{1}(t)$ is increasing in $t>0$, or equivalently if, $q_{i}(t)$ is $\mathrm{TP}_{2}(i, t)$ in $(i, t) \in\{1,2\} \times(0, \infty)$.

Example 1. Suppose that $X_{i}$ has an inverse Weibull distribution with distribution function

$$
F_{i}(x)=\exp \left\{-\left(\frac{\alpha_{i}}{x}\right)^{\beta_{i}}\right\}, \quad x>0, \alpha_{i}, \beta_{i}>0,
$$

and we shall denote it by $\operatorname{IW}\left(\alpha_{i}, \beta_{i}\right)$ for $i=1,2$. Then, it can be readily seen that $X_{1} \leqslant R A I X_{2}$ if and only if $\beta_{1} \geqslant \beta_{2}$.

Example 2 (The PRHR model). Suppose that $X_{i}$ has distribution function $F_{i}$ and suppose that $Y_{i}$ has distribution function $G_{i}$, for $i=1,2$. Let $G_{1}(x)=\left\{F_{1}(x)\right\}^{v_{1}}$ and let $G_{2}(x)=\left\{F_{2}(x)\right\}^{v_{2}}$, for $v_{1}>0$ and $v_{2}>0$. Then, it is easily verified that $X_{i} \leqslant_{R A I} Y_{i}$, for $i=1,2$. In parallel, it is easy to see that $X_{1} \leqslant R A I X_{2}$ if and only if $Y_{1} \leqslant R A I Y_{2}$.

Before stating another situation for application of the RAI order, we recall a semi-parametric family of distributions from Marshall and Olkin (2007). This family is called reversed hazard power (RHP) model. Formally, let $F(x \mid \theta)$ and $F(x)$ be such that $F(x \mid \theta)=\exp \left[-\{S(x)\}^{\theta}\right] ; \theta>0$, where $S(x)=-\ln F(x)$. Then the random variables $X_{\theta}$ and $X$ that have distributions $F(x \mid \theta)$ and $F(x)$, respectively, are said to satisfy in the RHP model.

Proposition 1. $X_{\theta_{1}} \leqslant R A I X_{\theta_{2}}$ if and only if $\theta_{1} \geqslant \theta_{2}$.

Proof. We have the identity

$$
F\left(x \mid \theta_{i}\right)=\exp \left[-\{S(x)\}^{\theta_{i}}\right]
$$

with $S(x)=-\ln F(x)$ and $i=1,2$. Note that $X_{\theta_{1}} \leqslant R A I X_{\theta_{2}}$ if and only if

$$
\frac{\ln F\left(x \mid \theta_{2}\right)}{\ln F\left(x \mid \theta_{1}\right)}=[S(x)]^{\theta_{2}-\theta_{1}}
$$

is increasing in $x$. Thus, the result follows if and only if $\theta_{1}>\theta_{2}$ since $S(x)$ is decreasing in $x$. 
Proposition 2. Let $X_{i}$ and $Y_{i}$ have distribution functions $F_{i}$ and $G_{i}$, respectively, for $i=1,2$. Let $G_{i}(x)=\exp \left[-\left\{S_{i}(x)\right\}^{\theta_{i}}\right]$ where $S_{i}(x)=$ $-\ln F_{i}(x), i=1,2$ and let $\theta_{1} \geqslant \theta_{2}$. Then

$$
X_{1} \leqslant{ }_{R A I} X_{2} \Rightarrow Y_{1} \leqslant{ }_{R A I} Y_{2} .
$$

Proof. By assumption $\left\{\frac{S_{2}(x)}{S_{1}(x)}\right\}^{\theta_{2}}$ is non-negative and increasing. Write

$$
\frac{\ln G_{2}(x)}{\ln G_{1}(x)}=\left\{\frac{S_{2}(x)}{S_{1}(x)}\right\}^{\theta_{2}} \cdot\left\{S_{1}(x)\right\}^{\theta_{2}-\theta_{1}} .
$$

Note that $S_{1}(x)$ is decreasing. Since $\theta_{1} \geqslant \theta_{2}$, then $\left\{S_{1}(x)\right\}^{\theta_{2}-\theta_{1}}$ is nonnegative and increasing. We can now conclude the proof.

In the following result, we demonstrate some implications among $\leqslant_{R A I}$ order and other well known stochastic orders and also we derive a preservation property under the DRHR aging class. Set $H(x)=\frac{\ln F_{2}(x)}{\ln F_{1}(x)}, x>0$ and let $f_{i}$ be the pdf of $X_{i}, i=1,2$.

By Definition $5, X_{1} \leqslant R A I X_{2}$ if and only if the function $S_{i}(x)=-\ln \left\{F_{i}(x)\right\}$ is $\operatorname{TP}_{2}(i, x)$ in $i \in\{1,2\}$ and $x \in(0, \infty)$. As a result, Theorem 2.1 in Rezaei et al. (2014) provides that $X_{1} \leqslant R R H X_{2}$ implies $X_{1} \leqslant R A I X_{2}$. In the literature, it is well known that $X_{1} \leqslant_{R H R} X_{2}$ implies $X_{1} \leqslant_{S T} X_{2}$ (cf. Shaked and Shanthikumar, 2007). One may wonder when does the reversed implication hold. The following theorem presents a condition for this to be hold.

Theorem 1. If $X_{1} \geqslant_{R A I} X_{2}$ and $X_{1} \leqslant_{S T} X_{2}$, then $X_{1} \leqslant_{R H R} X_{2}$.

Proof. Note that $X_{1} \geqslant_{R A I} X_{2}$ means that $H(x)=\frac{\ln F_{2}(x)}{\ln F_{1}(x)}$ is decreasing in $x>0$. It holds that

$$
H^{\prime}(x)=\frac{q_{2}(x) \ln F_{1}(x)-q_{1}(x) \ln F_{2}(x)}{\left\{\ln F_{1}(x)\right\}^{2}} .
$$

Thus, $X_{1} \geqslant_{R A I} X_{2}$ gives $H^{\prime}(x) \leqslant 0$, for all $x>0$. Observe that

$$
H^{\prime}(x) \leqslant 0, \forall x>0 \Leftrightarrow \frac{q_{2}(x)}{q_{1}(x)} \geqslant \frac{\ln F_{2}(x)}{\ln F_{1}(x)}, \forall x>0 .
$$

On the other hand, $X_{1} \leqslant_{S T} X_{2}$ provides that $\ln F_{2}(x) / \ln F_{1}(x) \geqslant 1$, for all $x>0$. So by the recent equivalence relation, we get $q_{2}(x) \geqslant q_{1}(x)$, for all $x>0$. That is $X_{1} \leqslant R H R X_{2}$. 
Theorem 2. The following assertions hold:

(i). If $X_{1} \geqslant_{R A I} X_{2}, H(x)$ is convex and $X_{2}$ is DRHR, then $X_{1}$ is DRHR.

(ii). If $0<\lim _{x \rightarrow 0^{+}} \frac{f_{2}(x)}{f_{1}(x)}<\infty$ and $X_{1} \leqslant R A I X_{2}$, then $X_{1} \leqslant S T X_{2}$.

(iii). If $\lim _{x \rightarrow \infty} \frac{f_{2}(x)}{f_{1}(x)} \leqslant 1$ and $X_{1} \leqslant R A I X_{2}$, then $X_{1} \geqslant_{R H R} X_{2}$.

Proof. (i). Take $S_{i}(x)=-\ln F_{i}(x)$ and notice that $S_{i}^{\prime}(x)=-q_{i}(x)$ for $i=1,2$ and $x \geqslant 0$. Also $H(x)=S_{2}(x) / S_{1}(x)$, for all $x>0$. The assumptions yield $H^{\prime}(x) \leqslant 0$ and $H^{\prime \prime}(x) \geqslant 0$ for all $x \geqslant 0$. Note that $X_{2} \in$ DRHR means that $S_{2}^{\prime \prime}(x) \geqslant 0$, for all $x \geqslant 0$, we then obtain

$$
\begin{aligned}
S_{1}^{\prime \prime}(x) & =\frac{d^{2}}{d x^{2}}\left(S_{2}(x) H(x)\right) \\
& =H(x) S_{2}^{\prime \prime}(x)+2 H^{\prime}(x) S_{2}^{\prime}(x)+H^{\prime \prime}(x) S_{2}(x),
\end{aligned}
$$

which is non-negative, for all $x \geqslant 0$, hence $X_{1} \in$ DRHR.

(ii). According to the definition, since $X_{1} \leqslant R A I X_{2}$ thus $H(x)$ is increasing and $F_{1}(0)=F_{2}(0)=0$. Therefore

$$
\begin{aligned}
H(x)= & \frac{\ln F_{2}(x)}{\ln F_{1}(x)} \\
& \geqslant \lim _{x \rightarrow 0^{+}}\left\{\frac{\ln F_{2}(x)}{\ln F_{1}(x)}\right\} \\
& =\lim _{x \rightarrow 0^{+}} \frac{f_{2}(x)}{f_{1}(x)} \times \frac{F_{1}(x)}{F_{2}(x)} \\
& =\left\{\lim _{x \rightarrow 0^{+}} \frac{f_{2}(x)}{f_{1}(x)}\right\}\left\{\lim _{x \rightarrow 0^{+}} \frac{F_{2}(x)}{F_{1}(x)}\right\}^{-1}=1
\end{aligned}
$$

Hence $H(x) \geqslant 1$ for all $x \geqslant 0$ and so $X_{1} \leqslant_{S T} X_{2}$. 
(iii). Note that $F_{i}(\infty)=1$ and as a result $\ln F_{i}(\infty)=0$, for $i=1,2$. We get, for all $x>0$

$$
\begin{aligned}
H(x)= & \frac{\ln F_{2}(x)}{\ln F_{1}(x)} \\
& \leqslant \lim _{x \rightarrow \infty} \frac{\ln F_{2}(x)}{\ln F_{1}(x)} \\
= & \lim _{x \rightarrow \infty} \frac{f_{2}(x)}{f_{1}(x)} \times \frac{F_{1}(x)}{F_{2}(x)} \\
= & \lim _{x \rightarrow \infty} \frac{f_{2}(x)}{f_{1}(x)} \leqslant 1 .
\end{aligned}
$$

Hence $\ln F_{1}(x) \leqslant \ln F_{2}(x)$, for all $x>0$, which is equivalent to $X_{1} \geqslant_{S T}$ $X_{2}$. Now, on using Theorem 1 , if $X_{1} \leqslant_{R A I} X_{2}$ and $X_{1} \geqslant_{S T} X_{2}$ hold, then $X_{1} \geqslant_{R H R} X_{2}$ holds.

The following example illustrates a situation where Theorem 2(i) is applicable.

Example 3. For a random variable $X$ with distribution $F$, suppose that $X_{\theta}$ has the RHP distribution $F_{\theta}(x)=\exp \left\{-S^{\theta}(x)\right\}$, for $x>0$, and $0<\theta<1$, where $S(x)=-\ln F(x)$. Let $X$ be DRHR. Since $H(x)=\ln F(x) / \ln F_{\theta}(x)=$ $S^{1-\theta}(x)$ is a decreasing convex function, thus Theorem 2(i) implies that $X_{\theta}$ is DRHR.

We present an example here to show that in Theorem 2(iii) the condition of $\lim _{x \rightarrow \infty} \frac{f_{2}(x)}{f_{1}(x)} \leqslant 1$ is necessary to conclude the result.

Example 4. Let $X_{1}$ and $X_{2}$ follow $\operatorname{IW}(1,2)$ and $\operatorname{IW}(1,1)$, respectively. Then, $F_{1}(x)=\exp \left[-x^{-2}\right]$ and $F_{2}(x)=\exp \left[-x^{-1}\right]$. For all $x>0$, we have

$$
\frac{f_{2}(x)}{f_{1}(x)}=\frac{x}{2} \exp \left[x^{-2}-x^{-1}\right]
$$

Thus $\lim _{x \rightarrow \infty} \frac{f_{2}(x)}{f_{1}(x)}=\infty$. That is the condition of Theorem 2(iii) does not hold. We know by Example 1 that $X_{1} \leqslant R A I X_{2}$. On the other hand, since

$$
\frac{F_{1}(x)}{F_{2}(x)}=\exp \left[-x^{-2}+x^{-1}\right]
$$


is not an increasing function in $x$ on $(0, \infty)$, hence $X_{1} \geqslant_{R H R} X_{2}$ fails to hold.

Theorem 3. If $\phi$ is strictly increasing, then $X_{1} \leqslant_{R A I} X_{2}$ implies $\phi\left(X_{1}\right) \leqslant R A I$ $\phi\left(X_{2}\right)$.

Proof. Let $T_{i}=\phi\left(X_{i}\right)$, for $i=1,2$ having distribution function $F_{T_{i}}$. Let $F_{i}$ be the distribution function of $X_{i}, i=1,2$. Then, we have the identity $-\ln \left\{F_{T_{i}}(x)\right\}=S_{i}\left(\phi^{-1}(x)\right), i=1,2$, where $S_{i}=-\ln \left(F_{i}\right), i=1,2$. The proof now obtains at once from the well known properties of totally positive of order 2 functions.

The following theorem is related with comparison of parallel systems according to $\leqslant R A I$ order. As we demonstrate in this result, the $\leqslant R A I$ order of components in two parallel systems is equivalent to the $\leqslant_{R A I}$ order among the lifetime of that parallel systems.

Theorem 4. Let $X_{i}, i=1,2, \ldots, n$ be i.i.d. from $F$ and let $Y_{i}, i=$ $1,2, \ldots, m$ be i.i.d. from $G$. Then, $X_{1} \leqslant R A I Y_{1}$ if and only if $\max \left\{X_{1}, \ldots, X_{n}\right\}$ $\leqslant_{R A I} \max \left\{Y_{1}, \ldots, Y_{m}\right\}$.

Proof. We know that, for all $x>0, P\left(\max \left\{X_{1}, \ldots, X_{n}\right\} \leqslant x\right)=F^{n}(x)$, and similarly $P\left(\max \left\{Y_{1}, \ldots, Y_{m}\right\} \leqslant x\right)=G^{m}(x)$. Hence, for any $x>0$, we have

$$
\frac{\ln P\left(\max \left\{Y_{1}, \ldots, Y_{m}\right\} \leqslant x\right)}{\ln P\left(\max \left\{X_{1}, \ldots, X_{n}\right\} \leqslant x\right)}=\frac{m}{n} \frac{\ln G(x)}{\ln F(x)} .
$$

By the definition, the above identity proves the equivalence relation in the theorem.

The following result considers the series systems. This result states that among two series systems, the one that its number of components is more ages faster than the other one in terms of the RAI order. Based on the HR ratio a similar result has been obtained in Theorem 1 of Lai and Xie (2003).

Theorem 5. Let $X_{1: m}$ and $X_{1: n}$ denote lifetimes of two series systems with $m$ and $n$ i.i.d. components, respectively. If $n>m \geq 1$, then $X_{1: n} \leqslant_{R A I} X_{1: m}$.

Proof. Take $q_{1: m}(x)$ and $q_{1: n}(x)$ to be the RHR functions of $X_{1: m}$ and $X_{1: n}$, respectively. Let each component of two systems have common distribution 
function $F$. To begin with, we take $m=n-1$. Then, we get

$$
\begin{aligned}
\frac{q_{1: m}(x)}{q_{1: n}(x)} & =\frac{d \ln \left\{1-\bar{F}^{m}(x)\right\} / d x}{d \ln \left\{1-\bar{F}^{n}(x)\right\} / d x} \\
& =\frac{n-1}{n} g\{\bar{F}(x)\},
\end{aligned}
$$

where $g:[0,1) \rightarrow[0, \infty)$, is defined as $g(x)=\left(1-x^{n}\right) /\left(x-x^{n}\right)$. It obtains that

$$
\frac{d}{d x}\left(\frac{x-x^{n}}{1-x^{n}}\right)=\frac{(1-x)\left(1+x+\cdots+x^{n-1}-n x^{n-1}\right)}{\left(1-x^{n}\right)^{2}},
$$

which is non-negative trivially for all $x \in[0,1)$. That is $g(x)$ is decreasing, concluding that $q_{1: m}(x) / q_{1: n}(x)$ is increasing in $x>0$. Hence, $X_{1: n} \leqslant R R H$ $X_{1: n-1}$. By continuing this procedure, we derive $X_{1: n} \leqslant R R H X_{1: n-1} \leqslant R R H$ $\cdots \leqslant R R H X_{1: m}$. That is to say $X_{1: n} \leqslant R R H X_{1: m}$. This further concludes that $X_{1: n} \leqslant R A I X_{1: m}$.

\section{Comparisons in Some Mixture Models}

In this section, we present some results for stochastic comparisons in some mixture models. The mixture PRHR model that was introduced earlier is taken into account. Furthermore, a new mixture model of the reversed hazard power is proposed here which is a possible extension of the RHP model. First we have the following result. It states that the random variable that has the mixture PRHR model is better than the baseline random variable in the model in the sense of the $\leqslant_{R A I}$ order.

Theorem 6. Let $X_{1}$ and $X_{2}$ have distribution functions $F_{1}$ and $F_{2}$, respectively, such that $F_{2}(x)=E\left\{F_{1}^{V}(x)\right\}, x \geqslant 0$. Then $X_{1} \leqslant_{R A I} X_{2}$.

Proof. We have

$$
\begin{aligned}
& \frac{d}{d x} \cdot \frac{\ln F_{2}(x)}{\ln F_{1}(x)}=\frac{d}{d x} \cdot \frac{\ln E\left\{F_{1}^{V}(x)\right\}}{\ln F_{1}(x)} \\
& \quad=\frac{q_{1}(x)}{\left\{\ln F_{1}(x)\right\}^{2} E\left\{F_{1}^{V}(x)\right\}}\left\{E\left(F_{1}^{V}(x)\left[\ln \left\{F_{1}^{V}(x)\right\}-\ln E\left\{F_{1}^{V}(x)\right\}\right]\right)\right\}
\end{aligned}
$$

Set $T=F^{V}(x)$ which is a non-negative random variable taking value on $[0,1]$, for all $x \geqslant 0$. Take $g(t)=t[\ln (t)-\ln \{E(T)\}], t \in[0,1]$. Obviously 
$g(t)$ is a convex function. Hence Jensen's inequality yields

$$
\begin{aligned}
E\left(F_{1}^{V}(x)\left[\ln F_{1}^{V}(x)-\ln E\left\{F_{1}^{V}(x)\right\}\right]\right)= & E\{g(T)\} \\
& \geqslant g\{E(T)\}=0 .
\end{aligned}
$$

The following result is a comparison between two mixture PRHR model.

Theorem 7. Let $\left(V_{1} \mid V=v\right) \leqslant_{S T}\left(V_{2} \mid V=v\right)$, for all $v \geqslant 0$, where $V=$ $V_{1}+V_{2}$ and assume that $V_{1}$ and $V_{2}$ are independent such that $E\left(V_{1}\right)=E\left(V_{2}\right)$. Then $X_{1} \leqslant R A I X_{2}$.

Proof. For each $i=1,2$, it obtains that

$$
\begin{aligned}
\frac{d}{d x} \ln E\left\{F_{0}^{V_{i}}(x)\right\} & =\frac{\frac{d}{d x} E\left\{F_{0}^{V_{i}}(x)\right\}}{E\left\{F_{0}^{V_{i}}(x)\right\}} \\
& =\frac{f_{0}(x) E\left\{V_{i} F_{0}^{V_{i}-1}(x)\right\}}{E\left\{F_{0}^{V_{i}}(x)\right\}}=q_{0}(x) \frac{E\left\{V_{i} F_{0}^{V_{i}}(x)\right\}}{E\left\{F_{0}^{V_{i}}(x)\right\}},
\end{aligned}
$$

where $q_{0}(x)=f_{0}(x) / F_{0}(x)$, which is the RHR of the baseline distribution $F_{0}$. Set $\left.S_{i}(x)=\ln E\left\{F_{0}(x)\right]\right\} V_{i}$, for $i=1,2$. Because $V_{1}$ and $V_{2}$ are, by assumption, independent thus

$$
\begin{aligned}
E\left\{F_{0}^{V_{1}}(x)\right\} E\left\{F_{0}^{V_{2}}(x)\right\} & =E\left\{F_{0}^{V_{1}}(x) F_{0}^{V_{2}}(x)\right\} \\
& =E\left\{F_{0}^{V_{1}+V_{2}}(x)\right\} \\
& =E\left\{F_{0}^{V}(x)\right\}, \quad \text { for any } x \geqslant 0,
\end{aligned}
$$

and also, for any $x \geqslant 0$, we have the identities

$$
E\left\{V_{2} F_{0}^{V_{2}}(x)\right\} E\left\{F_{0}^{V_{1}}(x)\right\}=E\left\{V_{2} F_{0}^{V_{1}+V_{2}}(x)\right\},
$$

and

$$
E\left\{V_{1} F_{0}^{V_{1}}(x)\right\} E\left\{F_{0}^{V_{2}}(x)\right\}=E\left\{V_{1} F_{0}^{V_{1}+V_{2}}(x)\right\}
$$


It suffices to show that the ratio $\ln E\left\{F_{0}^{V_{2}}(x)\right\} / \ln E\left\{F_{0}^{V_{2}}(x)\right\}$ is increasing in $x>0$. By using (3) we can get

$$
\begin{aligned}
\frac{d}{d x} \cdot \frac{\ln E\left\{F_{0}^{V_{2}}(x)\right\}}{\ln E\left(F_{0}^{V_{1}}(x)\right)} & =q_{0}(x) \frac{\frac{E\left\{V_{2} F_{0}^{V_{2}}(x)\right\}}{E\left\{F_{0}^{V_{2}}(x)\right\}} S_{1}(x)-\frac{E\left\{V_{1} F_{0}^{V_{1}}(x)\right\}}{E\left\{F_{0}^{V_{1}}(x)\right\}} S_{2}(x)}{S_{1}^{2}(x)} \\
& =\frac{q_{0}(x)}{S_{1}^{2}(x) E\left\{F_{0}^{V}(x)\right\}} E\left[F_{0}^{V_{1}+V_{2}}(x)\left\{V_{2} S_{1}(x)-V_{1} S_{2}(x)\right\}\right] .
\end{aligned}
$$

Since $\left(V_{1} \mid V=v\right) \leqslant_{S T}\left(V_{2} \mid V=v\right)$, for all $v \geqslant 0$, thus Theorem 1.A.3 (d) in Shaked and Shanthikumar (2007) concludes that $V_{1} \leqslant_{S T} V_{2}$. Because of Theorem 1.A.3 (a) in Shaked and Shanthikumar (2007), it follows that $\phi\left(V_{1}\right) \leqslant_{S T} \phi\left(V_{2}\right)$, for any decreasing function $\phi$, and consequently $E\left\{\phi\left(V_{1}\right)\right\} \geqslant E\left\{\phi\left(V_{1}\right)\right\}$, where the expectations exist. Fix $x>0$, and take $\phi(v)=F_{0}^{v}(x)$, which is decreasing in $v \geqslant 0$. Hence, $S_{1}(x)=\ln E\left\{\phi\left(V_{1}\right)\right\} \geqslant$ $\ln E\left\{\phi\left(V_{2}\right)\right\}=S_{2}(x)$, for all $x>0$. We know, by assumption, that $h(v)=$ $E\left(V_{2} \mid V=v\right)-E\left(V_{1} \mid V=v\right)$ is non-negative and that $E\{h(V)\}=0$. This implies that $P(h(V)=0)=1$, i.e.

$$
P\left\{E\left(V_{1} \mid V\right)=E\left(V_{2} \mid V\right)\right\}=1 .
$$

By conditioning on $V=V_{1}+V_{2}$, we, therefore, get

$$
\begin{aligned}
E\left[F_{0}^{V_{1}+V_{2}}(x)\left\{V_{2} S_{1}(x)-V_{1} S_{2}(x)\right\}\right] & =E\left[E\left(F_{0}^{V_{1}+V_{2}}(x)\left\{V_{2} S_{1}(x)-V_{1} S_{2}(x)\right\} \mid V\right)\right] \\
= & E\left[F_{0}^{V}(x)\left\{S_{1}(x) E\left(V_{2} \mid V\right)-S_{2}(x) E\left(V_{1} \mid V\right)\right\}\right] \\
= & \left\{S_{1}(x)-S_{2}(x)\right\} E\left\{F_{0}^{V}(x) E\left(V_{1} \mid V\right)\right\} \geqslant 0 .
\end{aligned}
$$

The proof is complete.

\section{Acknowledgement}

The authors are sincerely grateful to anonymous referees for their constructive comments and suggestions on the earlier version of this paper which lead to this improved version. This work was partially supported by the university of Birjand. 


\section{References}

Brito, G., Zequeira, R.I. and Valdés, J. E. (2011). On the Hazard Rate and Reversed Hazard Rate Orderings in Two-Component Series Systems with Active Redundancies. Statistics and Probability Letters, 81, 201-206.

Finkelstein, M. (2002). On the Reversed Hazard Rate. Reliability Engineering and System Safety, 78, 71-75.

Finkelstein, M. (2006). On Relative Ordering of Mean Residual Lifetime Functions. Statistics and Probability Letters, 76, 939-944.

Gupta, R.D. and Nanda, A.K. (2001). Some Results On Reversed Hazard Rate Ordering. Communications in Statistics-Theory and Methods, 30, 2447-2457.

Jiang, R., Ji, P. and Xiao, X. (2003). Aging Property of Univariate Failure Rate Models. Reliability Engineering and System Safety, 79, 113-116.

Kalashnikov, V.V. and Rachev, S.T. (1986). Characterization of Queuing Models and Their Stability. In: Prochorov, Y.K. (Ed.), Probability Theory and Mathematical Statistics, 2. Amsterdam, 37-53.

Lai, C.D. and Xie, M. (2003). Relative Ageing for Two Parallel Systems and Related Problems, Mathematical and Computer Modelling, 38, 1339-1345.

Li, X. and Li, Z. (2008). A Mixture Model of Proportional Reversed Hazard Rate. Communications in Statistics-Theory and Methods, 37, 2953-2963.

Li, X. and Zhao, P. (2011). On the Mixture of Proportional Odds Models. Communications in Statistics-Theory and Methods, 40, 333-344.

Marshall, A.W. and Olkin, I. (2007). Life Distributions. Springer, New York.

Nanda, A.K., Bhattacharjee, S. and Alam, S.S. (2007). Properties of Aging Intensity Function. Statistics and Probability Letters, 77, 365-373.

Nanda, A.K., Singh, H., Misra, N. and Paull, P. (2003). Reliability Properties of Reversed Residual Lifetime. Communications in Statistics-Theory and Methods, 32, 2031-2042.

Rezaei, M., Gholizadeh, B. and Izadkhah, S. (2014). On Relative Reversed Hazard Rate Order. Communications in Statistics - Theory and Methods, to appear.

Sengupta, D. and Deshpande, J.V. (1994). Some Results on the Relative Ageing of Two Life Distributions. Journal of Applied Probability, 31, 991-1003.

Shaked, M. and Shanthikumar, J.G. (2007). Stochastic Orders. Springer Verlag, New York. 
Xu, M. and Li, X. (2008). Negative Dependence in Frailty Models. J. Statist. Plann. Infer., 138, 1433-1441.

\section{Majid Rezaei}

Department of Statistics, School of Mathematical Sciences and Statistics,

University of Birjand, Birjand, Iran.

email: mjrezaei@birjand.ac.ir

\section{Vahideh Ahrari Khalaf}

Department of Statistics,

School of Mathematical Sciences

and Statistics,

University of Birjand,

Birjand, Iran.

email:v_ahrary84@yahoo.com 
